\title{
Dorsal Root Ganglion
}

National Cancer Institute

\section{Source}

National Cancer Institute. Dorsal Root Ganglion. NCI Thesaurus. Code C12462.

Gang lion with sensory function within the vertebral column. 